\title{
1 Title: Natural selection can favor ratchet robustness over mutational
}

\section{2 robustness}

\section{Authors:}

4 Yinghong Lan ${ }^{1 *}$, Aaron Trout ${ }^{2}$, Daniel Michael Weinreich ${ }^{1}$, Christopher Scott Wylie $^{1}$

\section{Institutional affiliations:}

6 1: Department of Ecology and Evolutionary Biology, and Center for Computational

7 Molecular Biology, Brown University, Providence, RI, USA

8 2: Department of Mathematics, Chatham University, Pittsburgh, PA, USA

\section{Corresponding author:}

$10 *$ : yinghong_lan@ brown.edu

\section{Abstract}

13 The vast majority of fitness-affecting mutations are deleterious. How natural populations evolve

14 to cope is a question of fundamental interest. Previous studies have reported the evolution of

15 mutational robustness, that is, natural selection favoring populations with less deleterious

16 mutations. By definition, mutational robustness provides a short-term fitness advantage.

17 However, this overlooks the fact that mutational robustness decreases finite asexual populations'

18 ability to purge recurrent deleterious mutations. Thus, mutational robustness also results in

19 higher risk of long-term extinction by Muller's ratchet. Here, we explore the tension between

20 short- and long- term response to deleterious mutations. We first show that populations can resist

21 the ratchet if either the selection coefficient or the ratio of beneficial to deleterious mutations

22 increases as fitness declines. We designate these properties as ratchet robustness, which 
23 fundamentally reflects a negative feedback between mutation rate and the tendency to

24 accumulate more mutations. We also find in simulations that populations can evolve ratchet

25 robustness when challenged by deleterious mutations. We conclude that mutational robustness

26 cannot be selected for in the long term, but it can be favored in the short-term, purely because of

27 temporary fitness advantage. We also discuss other potential causes of mutational robustness in

28 nature.

29 Keywords: natural selection; ratchet robustness; mutational robustness; fitness landscape;

30 Muller's ratchet.

\section{Introduction}

34 The vast majority of fitness-affecting mutations are deleterious. How natural populations evolve

35 to cope is a question of fundamental interest. One widespread hypothesis proposes the evolution

36 of mutational robustness, which would mean that deleterious mutations evolve to become less

37 deleterious(Krakauer and Plotkin 2001; Wilke and Adami 2003). Intuitively, it may seem that

38 organisms with higher mutational robustness will have a fitness advantage over those with lower

39 mutational robustness. Taking that intuition one step further, organisms with higher deleterious

40 mutation rate, i.e., more frequent occurrences of deleterious mutations, might be expected to

41 evolve greater mutational robustness. And indeed, there exists a substantial body of literature

42 consistent with that idea (Box 1).

43

44 Box 1: Literature on the evolution of mutational robustness 
45 Two computational studies (van Nimwegen et al. 1999; Wilke et al. 2001) have addressed the

46 possibility of evolution of mutational robustness. Using Avida, a platform for conducting in

47 silico evolutionary experiments (Ofria et al. 2009), Wilke et al. (2001) evolved populations

48 founded with the same ancestor under high and low mutation rates. Then they competed the two

49 evolved lineages against each other under different mutation rates. They found that the lineage

50 evolved under high mutation rate, albeit having lower fitness, wins under higher mutation rate.

51 Their interpretation was that that the lineage adapted to high mutation rate has higher mutation

52 robustness. Two experimental studies using a viroid (Codoner et al. 2006) or an RNA virus

53 (Sanjuan et al. 2007) similarly found that in competition at high mutation rate, populations

54 evolved at high mutation rate displaced those evolved at low mutation rate.

56 Working in the neutral network framework, van Nimwegen et al. (1999) explored a model in

57 which mutations are either neutral or lethal. In this case, mutational robustness of each genotype

58 is measured by its proportion of neutral mutations. They found that as mutation rate increases,

59 populations will evolve to be composed of genotypes with fewer lethal mutations, i.e., with

60 higher mutational robustness.

62 However, despite the intuitive appeal of the foregoing, it overlooks important components of

63 population genetics theory. Firstly, in infinite populations, where genetic drift can be neglected,

64 equilibrium mean fitness depends only on deleterious mutation rate (Haldane 1937; Kimura and

65 Maruyama 1966). Thus, mutational robustness, i.e., the reduced effect of deleterious mutations,

66 does not confer any long-term fitness advantage. Moreover, finite asexual populations risk

67 Muller's ratchet (Muller 1964; Haigh 1978), meaning that deleterious mutations accumulate at a 
rate so fast that selection is overwhelmed. In other words, besides the immediate fitness cost of

69 deleterious mutations, Muller's ratchet represents a longer-term challenge to evolving

70 populations. A previous study (Goyal et al. 2012) provides the quantitative parametric boundary

71 above which populations persist at Mutation-Selection-Drift Equilibrium (MSDE) (Wager and

72 Gabriel 1990; Schultz and Lynch 1997; Poon and Otto 2000), but below which they succumb to

73 the ratchet. In doing so, this study demonstrates that finite populations are less susceptible to

74 Muller's ratchet when mutation rate is lower, population size is greater, selection coefficient is

75 higher, or the ratio of beneficial to deleterious mutation rates is larger.

77 A fundamentally important question then arises as to how populations evolve in the face of both

78 short- and long- term challenges brought by deleterious mutations? Trivially, the evolution of

79 lower mutation rate will alleviate both concerns. Therefore, throughout this paper we hold

80 mutation rate as an extrinsic factor and observe other responses of evolving populations.

81 Furthermore, we hold population size constant, i.e., we assume soft selection. This approach is

82 conservative, because decreasing population size renders the population more prone to Muller's

83 ratchet, ramifications of which have already been studied in the context of mutational meltdown

84 (Lynch et al. 1993; Bull et al. 2005). Consequently, we focus only on the influence of selection

85 coefficient and the ratio of beneficial to deleterious mutation rates. As a complement to the

86 phrase "mutational robustness", we correspondingly designate increases in either of these

87 properties as conferring "ratchet robustness." (Recently Labar and Adami 2016 introduced the

88 phrase drift robustness for a similar phenomenon. However, we prefer ratchet robustness, since it

89 more precisely describes the underlying population genetic risk against which robustness is

90 conferred.) 
92 The fitness landscape provides a unifying framework for exploring both mutational robustness

93 and ratchet robustness. First define genotype space, where spatially adjacent genotypes are

94 mutationally adjacent, and then project the fitness of each genotype over the genotype space.

95 Since mutational robustness means deleterious mutations have smaller selection coefficients,

96 regions of the landscape with higher mutational robustness are "flatter". By contrast, "steeper"

97 regions of the landscape have higher ratchet robustness, since selection coefficients are larger.

98 These considerations seem to suggest an intrinsic tension between mutational robustness and

99 ratchet robustness. Ratchet robustness can also be realized by increased ratio of beneficial to

100 deleterious mutation rates, represented on the fitness landscape by the increased probability of

101 going "uphill" rather than "downhill" when locally exploring the genotype space via mutations

102 (Goyal et al. 2012).

103

104 The goal of this paper is to identify those features of the fitness landscape that are favored by

105 natural selection as a consequence of the fact that most mutations are deleterious. We investigate,

106 in isolation, the effect of selection coefficient, and the influence of ratio of beneficial to

107 deleterious mutation rates. We find that ratchet robustness protects populations from extinction

108 due to deleterious mutations and should therefore be favored in the long term, although in the

109 short term mutational robustness can also be selected for.

\section{Methods}

\section{Evolutionary model}


114 For all simulations, we implement discrete-time Wright-Fisher evolutionary model with custom

115 Python code. Within populations of fixed size $N$, each individual's genotype is solely identified

116 by the number of deleterious mutations it has, which is denoted by $i, i=0,1 \ldots, L$. $L$ represents

117 the genome length, which is also the maximum number of deleterious mutations possible.

118 Populations are represented by vectors $V$ of length $L+1$, where each element $V_{i}$ records the

119 number of individuals with $i$ deleterious mutations so that $\sum_{i} V_{i}=N$. Individuals with $i$

120 mutations have fitness denoted by

$$
W_{i}=e^{-s i^{1-\epsilon}}
$$

122 with selection coefficient $s$ and epistasis parameter $\epsilon . \epsilon=0$ in the absence of epistasis (Fig. 1), $\epsilon$

$123<0$ with negative epistasis (Fig. 2A \& B: $\epsilon=-0.25)$, and $\epsilon>0$ with positive epistasis (Fig. 2C \&

124 D: $\epsilon=0.25)$. Fitness defined by Eqn. 1 depends only on the number of deleterious mutations in

125 the genome and not their identity. We designate such fitness landscapes as isotropic.

127 Each generation of evolution consists of selection and mutation. The number of offspring with $i$

128 mutations is a random variable proportional to $V_{i}$, i.e., number of parents carrying $i$ mutations,

129 times $W_{i}$, i.e., fitness of these parents. Then, mutations are imposed by sampling from Poisson

130 distribution with parameter $U_{\text {del }}$ to generate number of deleterious mutations, and from Poisson

131 distribution with parameter $U_{b e n}$ to generate number of beneficial mutations. Finally, a new

132 vector $V^{\prime}$ is constructed, where $V_{i}^{\prime}$ records number of individuals with $i$ mutations in the new

133 generation. Simulations are performed for a prespecified number of generations, usually 10,000,

134 to ensure the result is unaffected by transient effects. We hold population size constant in all our

135 simulations, i.e., we assume soft selection, and characterize populations in which all individuals

136 are carrying $i=L$ deleterious alleles as being at extinction. 


\section{Infinite genome}

139 For the first part of the results, we hold constant the ratio of beneficial to deleterious mutation

140 rates $\left(U_{b e n} / U_{d e l}=0.01\right)$. Conceptually this is equivalent to the well-known infinite sites model

141 (Kimura 1969).

143 Simulations in Fig. 1 and Fig. 2 are performed on simple fitness landscapes with $L=300$, with

144 only one isotropic peak defined by Eqn. 1.

146 We characterize the critical $U_{d e l}$, i.e., the highest $U_{d e l}$ under which populations can resist

147 Muller's ratchet with reference to the time variance in fitness. In Fig. S2, we present the variance

148 in fitness across time at equilibrium for the simulations conducted in Fig. 1A. Notably, the

149 critical $U_{d e l}$, i.e., the highest $U_{d e l}$ under which populations can resist Muller's ratchet is seen to

150 overlap with the $U_{d e l}$ under which time variance in fitness reaches maximum. This is reasonable,

151 because when $U_{d e l}$ is well below or well above the critical $U_{d e l}$, populations are either at MSDE

152 or extinction and thus exhibit low time variance in fitness. In contrast, near the critical $U_{d e l}$, the

153 "tug-of-war" between the two possible states maximizes stochasticity.

155 We utilize the above observation to approximately locate the critical $U_{d e l}$ in Fig. 1B, across

156 different population size $N$ and selection coefficient $s$. For each $N$ and $s$ combination, we record

157 the time variance in fitness under different $U_{d e l}$, and report the $U_{d e l}$ associated with highest time

158 variance as the critical $U_{d e l}$. There are three sources of uncertainty in this analysis. First, it is

159 impossible to conduct simulations under every possible $U_{d e l}$ value. In practice, we sample $U_{d e l}$ 
160 at intervals of 0.025 , meaning that the "true" critical $U_{d e l}$ may fall in between two adjacent

161 sampled $U_{d e l}$ values. Second, because of this intrinsic granularity, the detected maximum time

162 variance may not be the true maximum. We measure such uncertainty as the range of $U_{d e l}$ values

163 that show time variance above half of the detected maximum. Third, there is inevitable variation

164 in recorded fitness variance across replicated simulations, due to the stochastic nature of the

165 simulation. However, we find that the second source of variation dominates the other two by at

166 least one order of magnitude (not shown). Therefore, we portray only the second type of

167 uncertainty as error bars in Fig. 1B.

168

169 For simulations in Fig. 3, as shown in Fig. 3A, we fuse two isotropic peaks together: on the

170 positive epistasis side of the intervening fitness valley, $\epsilon=0.25$ and $L=270$; on the negative

171 epistasis side, $\epsilon=-0.25$ and $L=29$. (These values of $L$ were chosen so that fitness values on both

172 sides of the floor of the valley would match.) Mutation on both sides of the valley were biased as

173 described above, i.e. $U_{b e n} / U_{\text {del }}=0.01$.

175 For simulations in Fig. 4, we define a hybrid fitness landscape consisting of two domains, one

176 exhibiting positive epistasis and the other exhibiting negative epistasis. As shown in Fig. 4A, a

177 fraction $p$ of the first mutations put individuals in the positive epistasis domain, where $\epsilon=0.25$

178 and $L=300$, while the remaining fraction $1-p$ of first mutations put individuals at the negative

179 epistasis domain where $\epsilon=-0.25$ and $L=300$. If a lineage returns to the peak (by beneficial

180 mutations), the same $p$ and $1-p$ apply, i.e., lineages can "travel" between the two domains

181 through the peak. 


\section{Finite genome}

184 For the second part of the results, we allow $U_{b e n} / U_{\text {del }}$ to evolve with the proportion of

185 deleterious mutation in the genome. Specifically, $U_{b e n}$ equals proportion of deleterious

186 mutations times the total mutation rate $U$, while $U_{d e l}$ equals $U-U_{b e n}$. Consequently, $U_{b e n}$ and

$187 U_{d e l}$ are unique for individuals in different $V_{i}$, and we can only specify total mutation rate. In Fig.

1885 , simulations are conducted on an isotropic non-epistatic fitness landscape with $L=10$ and $s=$ $189 \quad 0.4$

\section{Results}

193 We use individual-based simulations of finite populations of asexual organisms to study the

194 ability of selection and evolving ratios of beneficial to deleterious mutation rates to protect

195 populations from deleterious mutations. We first assume a constant ratio of beneficial to

196 deleterious mutation rates and examine evolutionary behavior as a function of fitness landscape

197 topography. We then allow the ratio of beneficial to deleterious mutation rates itself to evolve

198 while holding other aspects of the fitness landscape constant.

200 Effect of fitness landscape topology

201 We simulate evolution on four kinds of fitness landscapes with increasing complexity, while

202 holding the ratio of beneficial to deleterious mutation $\left(U_{b e n} / U_{d e l}\right)$ equal to 0.01 . We define a

203 fitness peak as isotropic if the fitness of any genotype only depends on its number of deleterious

204 mutations, but not the identity of these mutations. We start by examining evolutionary behavior 
on fitness landscapes with a single peak where every deleterious mutation shares the same effect

206 regardless of the current genome (Eqn. 1: $\epsilon=0$, Fig. 1A inset). This is, by definition, an

207 isotropic peak in the absence of epistasis. Next, we allow the effect of deleterious mutations to

208 increase (Eqn. 1: $\epsilon<$ 0, Fig. 2A) or decrease (Eqn. 1: $\epsilon>0$, Fig. 2C) with the number of

209 deleterious mutations in the current genome. In other words, we are adding epistasis to the

210 isotropic peak, distinguishing between negative (Fig. 2A) and positive (Fig. 2C) epistasis. We

211 then examine evolutionary behavior on fitness landscapes consisting of mutationally adjacent

212 isotropic peaks with negative and positive epistasis (Fig. 3A). Finally, we relax our assumption

213 of isotropic peaks, and model populations evolving on hybrid fitness peaks characterized by

214 domains of positive and negative epistasis (Fig. 4A).

215

216 In the absence of epistasis, populations residing on steeper fitness landscapes survive under

\section{7 higher deleterious mutation rate}

218 We first confirm the effect that stronger selection is better able to protect populations from

219 Muller's ratchet, or equivalently, that mutational robustness can enable the ratchet (Goyal et al.

220 2012). To do so, we construct two fitness landscapes, each composed of one isotropic peak but

221 with different steepness (selection coefficients $s$ ) in the absence of epistasis (Fig. 1A inset). We

222 simulate the evolution of finite asexual populations on such landscapes under different

223 deleterious mutation rates, and record their fitness at equilibrium (Fig. 1A). As mentioned above,

224 we implement soft selection, and we define extinction as occurring when all individuals in the

225 population attain the minimum possible fitness. 
227 Under low mutation rates, selection is able to purify deleterious mutations, and drift (and thus,

228 the ratchet) is irrelevant on both flat and steep landscapes. This is because the stochastic loss of

229 fittest class is extremely rare: purifying selection is strong relative to mutation, and if lost, the

230 fittest class is quickly restored by beneficial mutations. Consequently, equilibrium fitness lies

231 close to the well-known infinite population expectation $e^{-U_{d e l}}$ (black dashed line in Fig. 1A,

232 Haldane 1937; Kimura and Maruyama 1966). However, after $U_{\text {del }}$ exceeds some critical value

233 (at this population size, $U_{d e l} \approx 0.4$ for $s=0.1$ and $U_{d e l} \approx 1.9$ for $s=0.4$, see Fig. 1A), rapidly

234 accumulating deleterious mutations overwhelm selection, leading to extinction by Muller's

235 ratchet. We name the highest $U_{\text {del }}$ under which populations are able to resist Muller's ratchet the

236 "critical $U_{d e l}$ ". Our simulation results for the critical $U_{d e l}$ under different $N$ and $s$ are consistent

237 with analytical results based on Eqn. 7 in Goyal et al. 2012 (see Fig. 1B). Given a fixed

238 population size $(N)$ and constant $U_{b e n} / U_{d e l}$, populations on steeper landscapes are able to

239 survive under higher $U_{d e l}$, i.e., they demonstrate a higher critical $U_{d e l}$ (Fig. 1A \& B, Goyal et al.

240 2012). In other words, in the long term, increased mutational robustness increases susceptibility

241 to Muller's ratchet.

243 Fitness landscapes with negative epistasis protect populations from Muller's ratchet while

244 ones with positive epistasis make populations more vulnerable to the ratchet

245 Epistasis means the dependence of mutational fitness effects on the genetic background. Without

246 epistasis (Fig. 1), there is no evolution of selection coefficients, since each mutation will always

247 have the same effect. Put differently, epistasis determines how selection changes as deleterious

248 mutations accumulate, shaping both local mutational and ratchet robustness experienced by an

249 evolving population. In order to understand the role of epistasis, we consider the two simplest 
cases: one where selection strength monotonically increases with the number of deleterious

251 mutations, and the other where selection strength monotonically decreases with the number of

252 deleterious mutations. In other words, we consider a single isotropic peak with either only

253 negative epistasis (Fig. 2A) or only positive epistasis (Fig. 2C), respectively.

255 When epistasis is negative, as deleterious mutations accumulate, the local strength of purifying

256 selection increases, and consequently subsequent deleterious mutations become less likely to

257 accumulate. This hints at a negative feedback between the accumulation of deleterious mutations

258 and the tendency to accumulate more, which potentially could halt Muller's rachet (as previously

259 seen in Kondrashov 1994). On the other hand, when epistasis is positive, as deleterious

260 mutations accumulate, local selection weakens, and deleterious mutations can more easily

261 accumulate. This suggests a positive feedback between the aforementioned two forces, which

262 may render populations more vulnerable to the ratchet than those examined above. Importantly,

263 the critical $U_{d e l}$ will thus vary across the fitness landscape in response to this variation in the

264 strength of selection

266 Our simulations are consistent with this intuition (Fig. 2B, Fig. 2D). In the presence of negative

267 epistasis, when $U_{d e l}$ is lower than the critical $U_{d e l}$ at the peak, it is lower than the critical $U_{d e l}$

268 anywhere on the landscape. Therefore, selection will drive the population back toward the peak

269 regardless of where on the landscape it is initialized (Fig. 2B). Even if $U_{d e l}$ is higher than the

270 critical $U_{d e l}$ at the peak, there exists a point on the landscape where selection exactly offsets such

$271 U_{d e l}$, because purifying selection increases monotonically from the peak (see also S1 Text). If a

272 population is initialized below this point, selection locally will be strong enough to push 
273 populations upward until this point is reached. If instead, a population is initialized above this

274 point, mutation will be strong enough to push the population downward to this point, but no

275 further. Therefore, even under high $U_{d e l}$, landscapes with negative epistasis will halt Muller's

276 ratchet and establish stable MSDE.

278 Conversely, on the landscape with positive epistasis, when $U_{d e l}$ is lower than the critical $U_{d e l}$ at

279 the peak, populations initiated at the peak can maintain MSDE. However, positive epistasis

280 means that purifying selection decreases monotonically from the peak, and consequently there

281 exists a point on the landscape where selection exactly offsets such $U_{d e l}$. Populations initiated

282 above this point will experience selection stronger than required to offset $U_{d e l}$ and evolve to the

283 peak, while populations initiated below this point suffer from selection weaker than needed to

284 offset $U_{d e l}$, and will succumb to Muller's ratchet (Fig. 2D). Therefore, we refer to this point as

285 "point of no return". Importantly, even if parameter values are such that populations can

286 equilibrate at the peak, stochastic fluctuations will eventually take them across this point of no

287 return, after which they will succumb to the ratchet. Moreover, as $U_{d e l}$ increases, the selection

288 required to oppose mutation naturally increases as well. Correspondingly, as $U_{d e l}$ increases, the

289 point of no return migrates towards the peak. This imposes a greater danger of succumbing to

290 Muller's ratchet for populations in the vicinity of the peak via stochastic fluctuations. Finally,

291 once $U_{d e l}$ is greater than the critical $U_{d e l}$ at the peak, the point of no return overlaps the peak,

292 and mutation overwhelms selection everywhere on the fitness landscape. 
295 We next demonstrate that populations can indeed evolve to occupy regions of the fitness

296 landscape with negative epistasis when challenged by deleterious mutations. To do so, we

297 construct a fitness landscape composed of two mutationally adjacent isotropic peaks featuring

298 opposite signs of epistasis (Fig. 3A). Populations finding themselves below the point of no return

299 on the positive epistasis side will experience selection weaker than needed to offset mutation and

300 their fitness will decline to the valley, similar to populations declining to the bottom of the

301 landscape in Fig. 2D. Moreover, populations above the point of no return will nevertheless

302 experience stochastic fluctuations and will be eventually carried over the point of no return, at

303 which point they will also evolve to the valley. However, upon arrival at the valley, strongly

304 beneficial mutations become available, drawing populations onto the negative epistasis side of

305 the valley, after which they quickly climb to MSDE (Fig. 3B). (Note that $U_{b e n}=0.01 U_{d e l}$ on

306 both sides of the valley, and consequently this behavior is driven entirely by differences in the

307 local strength of natural selection.) Since the two peaks share identical selection coefficients at

308 the peak, the positive epistasis side has uniformly higher mutational robustness but uniformly

309 lower ratchet robustness. This extends our understanding of the tension between mutational and

310 ratchet robustness and demonstrates that ratchet robustness, instead of mutational robustness, is

311 likely to evolve in response to high deleterious mutation rates.

\section{Mutational robustness and ratchet robustness on hybrid peaks}

314 Biologically realistic landscape peaks are unlikely to have only negative or positive epistasis. As

315 a first attempt to investigate the complexity introduced by heterogeneity in epistasis, we now

316 allow the sign of epistatic effects of mutations to be dependent on the first mutation away from

317 the peak. Specifically, a certain fraction $(p)$ of the first mutations now place the evolving 
318 population on a region of the landscape exhibiting positive epistasis, while the remaining fraction

$319(1-p)$ place the evolving population on a region of the landscape exhibiting negative epistasis

320 (Fig. 4A). Concretely, among all the mutational paths leaving the peak, a proportion $p$ of them

321 show positive epistasis, while the remaining fraction $1-p$ show negative epistasis. We assume

322 that all first mutations share identical fitness effects, so that there is no immediate fitness

323 advantage to lineages entering the region with negative or positive epistasis. However, for all

324 genotypes with greater than one deleterious mutation, fitness is necessarily higher in regions of

325 the landscape with positive epistasis than in regions with negative epistasis (Eqn. 1). In other

326 words, regions of the landscape with positive epistasis have both higher fitness and higher

327 mutational robustness (but lower ratchet robustness) compared with ones with negative epistasis.

328 Note that the only mutational path between regions is through the peak. We evolved populations

329 on such hybrid peak with $p=0.5$ and report the proportions of populations residing exactly at

330 the peak, in the negative epistasis region, and in the positive epistasis region as a function of $U_{d e l}$

331 (Fig. 4B).

333 When $U_{d e l}$ is so low that majority of the individuals carry zero or one mutation (here $U_{d e l} \approx 0.2$,

334 Fig. 4B, Fig. S3A), the proportion of the population on the negative epistasis region is very close

335 to that on the positive epistasis region. This merely reflects the fact that $p$ equals 0.5 , since the

336 fitness cost of the first mutation is the same. However, as $U_{d e l}$ rises to moderate level (here $\sim 0.2$

$337<U_{d e l}<\sim 1.1$, Fig. 4B, Fig. S3B), the proportion of the population on the negative epistasis

338 region begins to drop below 0.5 . This reflects the selective enrichment of the subpopulation

339 experiencing positive epistasis: individuals with two or more mutations on the positive epistasis

340 region are fitter than are those with the same number of mutations on the negative epistasis 
341 region. Nevertheless, in this intermediate range of values of $U_{d e l}$, a subpopulation on the

342 negative epistasis region is still sustained despite lower fitness, thanks to net mutational inflow

343 from the subpopulation at the peak (Table S1). In essence, the subpopulation on the negative

344 epistasis region is at mutation-selection balance: constantly being purified by selection but being

345 regenerated by mutation from the peak.

347 However, after $U_{d e l}$ increases enough that the peak can no longer be sustained (here, $U_{d e l} \approx 1.1$ ),

348 the proportion of the population at the peak become negligible (Fig. 4B, Fig. S3C). As a result,

349 the subpopulation residing on the negative epistasis region of the fitness landscape is

350 mutationally disconnected from the peak and is quickly wiped out by selection. The remaining

351 population now occupies only the positive epistasis region. However, since $U_{d e l}$ has

352 overwhelmed selection at the peak, it necessarily does so also at every other point in the positive

353 epistasis region, and the population quickly succumbs to Muller's ratchet. This threshold

354 recapitulates results seen when the point of no return reaches the peak on isotropic fitness peaks

355 with positive epistasis region (Fig. 2C \& 2D, although the numeric value of the critical mutation

356 rate differs here, reflecting its dependence on the fitness landscape).

358 More importantly, the observed pattern does not depend on the particular value $p=0.5$ : even

359 when there is only a very small fraction of paths leaving the peak with positive epistasis,

360 subpopulations on the positive epistasis region of the landscape will be favored due to their

361 short-term fitness advantage (Fig. 4C). Such an advantage is amplified by higher $U_{d e l}$, so long as

362 it remains less than the critical $U_{d e l}$ at the peak, i.e., the $U_{d e l}$ under which individuals at the peak

363 are lost to Muller's ratchet. Below this threshold, the two subpopulations accumulate more 
364 mutations, and thus experience increased fitness differences. Eventually, once $U_{d e l}$ exceeds the

365 critical $U_{d e l}$ at the peak, the mutational connection between subpopulations on the fitness

366 landscape is again extinguished. At this point both subpopulations are doomed. The lower-fitness

367 subpopulation on the region of the landscape with negative epistasis will lose its mutational input

368 and go extinct. At the same time, the remaining higher-fitness subpopulation on the region with

369 positive epistasis will succumb to Muller's ratchet.

\section{Effect of ratio of beneficial to deleterious mutation rates}

372 In order to isolate the role that topographic features of the fitness landscape play in an evolving

373 population's response to deleterious mutations, we have thus far assumed that the ratio of

374 beneficial to deleterious mutation rates remains fixed (see Methods). However, as deleterious

375 mutations accumulate, this ratio can itself evolve, due to reversion mutations. Moreover,

376 compensatory mutations (mutations that are only beneficial in the presence of deleterious

377 mutations at other loci) amplify this effect. For example, one survey estimated that each

378 deleterious mutation gives rise to approximately 12 compensatory mutations (Poon and Chao

$3792005)$.

381 Increasing ratio of beneficial to deleterious mutation rates with decreasing fitness protects

382 populations from Muller's ratchet

383 We therefore modify our simulations to allow deleterious mutations to revert, thereby causing

$384 U_{b e n} / U_{d e l}$ to increase with the accumulation of deleterious mutations. Biologically, this

385 approach is conservative because it neglects compensatory mutations induced by deleterious

386 mutation (e.g., Poon and Chao 2005). Since increasing $U_{b e n} / U_{d e l}$ reduces the rate at which new 
deleterious mutations occur, this suggests that another negative feedback may exist between the

388 accumulation of deleterious mutations and the tendency to accumulate more (Goyal et al. 2012).

389 This is reminiscent of the one due to negative epistasis seen above (Fig. 2A). To gain more

390 insight into this effect, we compare equilibrium fitness in populations evolved when $U_{b e n} / U_{\text {del }}$

391 is allowed to change with that seen in populations evolved under fixed $U_{b e n} / U_{d e l}$ (Fig. 5). As $U$

392 increases and deleterious mutations accumulate, we see that populations with varying $U_{\text {ben }} / U_{\text {del }}$

393 enjoy the benefit of lower $U_{d e l}$ and equilibrate at higher fitness than do populations with fixed

$394 U_{b e n} / U_{d e l}$. Eventually, under sufficiently high $U$, populations with fixed $U_{b e n} / U_{d e l}$ succumb to

395 Muller's ratchet, as seen above. In contrast, populations with varying $U_{b e n} / U_{d e l}$ halts the ratchet,

396 as previously described (Goyal et al. 2012).

399 Discussion

400 Negative feedbacks halt Muller's ratchet

401 Our results highlight the importance of negative feedbacks in halting Muller's ratchet. The

402 mechanisms responsible for such negative feedback may be varied, realized via negative

403 epistasis (Fig. 2 \& 3), or increasing $U_{b e n} / U_{\text {del }}$ (Fig. 5). We note that many surveys of biological

404 fitness landscapes find extensive evidence for negative epistasis (Bershtein et al. 2006; Costanzo

405 et al. 2010; Khan et al. 2011; Steinberg and Ostermeier 2016). Additionally, $U_{b e n} / U_{d e l}$ tends to

406 increase as deleterious mutations accumulate due to reversions and compensatory mutations.

407 More importantly, both negative epistasis and increasing $U_{b e n} / U_{d e l}$ can be seen as representative

408 cases of changing distribution of fitness effects (DFE) (Eyre-Walker and Keightley 2007) with

409 decreasing fitness. Negative epistasis influences the magnitude of mutational effects, while 
410 increasing $U_{b e n} / U_{d e l}$ influences the ratio between beneficial and deleterious mutations. While

411 outside the scope of our simulations, we suggest that as fitness goes down, both aspects of the

412 DFE can simultaneously evolve, resulting in a wide range of potential DFE changes that confer

413 ratchet robustness.

415 In fact, even in the presence of positive epistasis, it's plausible that increases in $U_{b e n} / U_{d e l}$ could

416 still halt Muller's ratchet. Conversely, even if $U_{b e n} / U_{d e l}$ decreases as deleterious mutations

417 accumulate, sufficiently strong negative epistasis could in principle protect populations from

418 deleterious mutations. (Although as noted above, there is little reason to believe that $U_{\text {ben }} / U_{\text {del }}$

419 should decline with fitness.) At this moment, we remain ignorant about the general rules driving

420 changing DFE as a function of fitness. In the future, theoretical models that parameterize the

421 DFE could be built to quantify the relative importance of negative epistasis and increasing

$422 U_{b e n} / U_{d e l}$ in protecting evolving populations from deleterious mutation. Experimental studies

423 could also be conducted to compare DFE at different fitness levels for various biological systems.

424 Notably, a previous experimental study using bacteriophage has observed that as fitness declines,

425 the rate of beneficial mutations changes much more drastically than does the size of mutational

426 effects (Silander et al. 2007).

428 Widespread existence of mutational robustness may result from selection for

429 environmental robustness 
432 On close inspection, Fig. 2 in Wilke et al. 2001 (reproduced here as Fig. 6) may suggest the

433 evolution of ratchet robustness rather than of mutational robustness. Specifically, the fitness

434 distribution of genotypes in the mutational neighborhood of the lineage adapted under high

435 mutation rate appears to contain more lethal mutations than do those mutationally adjacent to the

436 lineage adapted to low mutation rate. Furthermore, the lineage adapted under low mutation rate

437 seems more prone to mutation accumulation than does the lineage adapted under high mutation

438 rate. Both observations suggest that the lineage adapted under low mutation rate experiences

439 weaker purifying selection than does the one adapted under high mutation rate. Thus, we

440 interpret the data in Wilke et al. to suggest that the lineage adapted under high mutation rate

441 actually has lower mutational robustness, and consequently higher ratchet robustness, consistent

442 with our results.

443

444 Unfortunately, the two studies (Codoner et al. 2006; Sanjuan et al. 2007) attempting to

445 experimentally validate conclusions in Wilke et al. failed to fully characterize the different

446 lineages' fitness landscapes. Specifically, only non-lethal genotypes were considered, while

447 lethal mutations were ignored. Thus, we are unable to assess whether the lineage evolved under

448 high mutation rate has higher mutational robustness than the lineage evolved under low mutation

449 rate, since only a fraction of the DFE was measured.

450

451 Findings in van Nimwegen et al. (1999) are actually in agreement with our conclusions here, as

452 previously noted (Wylie and Shakhnovich 2011). Specifically, by construction Muller's ratchet

453 is impossible in van Nimwegen et al.'s model, since any non-neutral mutation is lethal and so is

454 instantaneously eliminated. Put another way, under this model evolving populations enjoy the 
456 succumbing to Muller's ratchet. Relaxing van Nimwegen et al.'s assumption of strict lethality for

457 all non-neutral mutations recovers exactly our predicted behavior: at equilibrium, mutational

458 robustness declines with mutation rate (see Fig S5 in Wylie and Shakhnovich 2011). The same

459 argument also applies to other studies where all deleterious mutations are lethal, e.g., Wilke 2001.

461 Our results suggest that mutational robustness cannot be selected for the long term, and we

462 address previous theoretical studies on the evolution of mutational robustness in Box

463 2. Nevertheless, mutational robustness is seen at many levels of biological systems (Wagner

464 2013). We note however that the existence of mutational robustness need not imply selection for

465 mutational robustness (Hermisson and Wagner 2004; Proulx and Phillips 2005; Siegal and Leu

466 2014). Following others (de Visser et al. 2003), we suggest instead that mutational robustness

467 may often evolve as a correlated consequence of selection for environmental robustness, i.e., an

468 organism's ability to sustain fitness against environmental perturbations. Theoretically and

469 empirically, environmental robustness has been shown to give rise to mutational robustness

470 (Wagner et al. 1997; Ancel and Fontana 2000; Meiklejohn and Hartl 2002; de Visser et al. 2003;

471 Szollosi and Derenyi 2007; Goldsmith and Tawfik 2009; Domingo-Calap et al. 2010).

473 Why can natural selection favor the evolution of environmental but not mutational robustness?

474 The key distinction is that mutational robustness requires tolerating heritable perturbations,

475 which inevitably alters the "starting point" of future generations. Such heritable decay is intrinsic

476 to Muller's ratchet. By contrast, selection for environmental robustness entails non-heritable

477 environmental perturbation. Consequently, the short-term advantage of environmental robustness 
478 is not offset by any long-term cost, accounting for the absence of an "environmental ratchet". In

479 summary, while mutational robustness may be widespread in nature, we suggest one alternative

480 interpretation for its evolution: namely as a correlated consequence of selection for

481 environmental robustness (de Visser et al. 2003). We address another possibility next.

\section{Long-term and short-term fate of mutational and ratchet robustness}

484 Our findings also illustrate that mutational robustness can be selected for in the short term,

485 despite the danger it imposes in the long term (Fig. 4). This suggests another mechanism for the

486 existence of mutational robustness in natural populations. How populations overcome this

487 shortsightedness remains an open question. One possibility described in a previous study

488 (O'Fallon et al. 2007) is that subdivision can protect populations from myopic selection for

489 mutational robustness. Because selection is more effective at purging deleterious mutations in

490 demes dominated by ratchet robust individuals, in that study net dispersal rates were higher from

491 those demes, and the population in total was thus enriched for such individuals in spite of the

492 short-term disadvantage. We predict that any population structure capable of hindering rapid

493 fixation of mutational robustness will help natural selection favor ratchet robustness. However, a

494 detailed survey of possible mechanisms is outside the scope of this study.

496 Conclusion

497 It is of fundamental interest to understand how natural populations evolve to cope with the fact

498 that almost all mutations are deleterious. Intuitively, natural selection may favor mutational

499 robustness, as deleterious mutations of lesser effects seem to pose less danger. However, we

500 have extended work of others to demonstrate another peril of deleterious mutations: long term 
extinction via Muller's ratchet. Indeed, we show that mutational robustness increases the risk of

Muller's ratchet. We then demonstrate that both negative epistasis and increasing $U_{b e n} / U_{d e l}$

confer ratchet robustness and can protect populations from deleterious mutations in the long term.

504

505

506

507

508

509

510

511

512

513

514

515

516

517

518

519

520

521

522

523

524

525

526

527

528

529

530

531

532

533

534

535

536

537

538
Overall, our findings indicate that natural selection can favor ratchet robustness over mutational

robustness, and we define important areas of future theoretical and experimental work.

\section{References}

Ancel, L. W., and W. Fontana. 2000. Plasticity, Evolvability, and Modularity in RNA. J. Exp. Zool. 288:242-283.

Bershtein, S., M. Segal, R. Bekerman, N. Tokuriki, and D. S. Tawfik. 2006. Robustness-epistasis link shapes the fitness landscape of a randomly drifting protein. Nature 444:929-932.

Bull, J. J., L. A. Meyers, and M. Lachmann. 2005. Quasispecies made simple.

Codoner, F. M., J.-A. Daros, R. V. Sole, and S. F. Elena. 2006. The Fittest versus the Flattest $\square$ : Experimental Confirmation of the Quasispecies Effect with Subviral Pathogens. Plos Pathog. 2:e136.

Costanzo, M., A. Baryshnikova, J. Bellay, Y. Kim, E. D. Spear, C. S. Sevier, H. Ding, J. L. Y. Koh, K. Toufighi, S. Mostafavi, J. Prinz, R. P. St Onge, B. VanderSluis, T. Makhnevych, F. J. Vizeacoumar, S. Alizadeh, S. Bahr, R. L. Brost, Y. Chen, M. Cokol, R. Deshpande, Z. Li, Z.-Y. Lin, W. Liang, M. Marback, J. Paw, B.-J. San Luis, E. Shuteriqi, A. H. Y. Tong, N. van Dyk, I. M. Wallace, J. A. Whitney, M. T. Weirauch, G. Zhong, H. Zhu, W. A. Houry, M. Brudno, S. Ragibizadeh, B. Papp, C. Pál, F. P. Roth, G. Giaever, C. Nislow, O. G. Troyanskaya, H. Bussey, G. D. Bader, A.-C. Gingras, Q. D. Morris, P. M. Kim, C. A. Kaiser, C. L. Myers, B. J. Andrews, C. Boone, S. J. Dixon, M. Costanzo, A. Baryshnikova, B. Andrews, C. Boone, L. Hartwell, A. H. Tong, A. H. Tong, R. Mani, R. P. St. Onge, J. L. Hartman, G. Giaever, F. P. Roth, R. P. S. Onge, D. Segrè, A. Deluna, G. M. Church, R. Kishony, A. Huber, E. J. Chen, C. A. Kaiser, M. C. Jonikas, M. B. Metzger, S. Michaelis, S. Leidel, P. B. Rahl, C. Z. Chen, R. N. Collins, A. Esberg, B. Huang, M. J. Johansson, A. S. Byström, T. Naumanen, L. D. Johansen, E. T. Coffey, T. Kallunki, L. D. Johansen, S. F. Levy, M. L. Siegal, A. Levchenko, P. M. Kim, L. J. Lu, Y. Xia, M. B. Gerstein, H. B. Fraser, D. P. Wall, A. E. Hirsh, C. Pál, B. Papp, L. D. Hurst, P. M. Kim, A. Sboner, Y. Xia, M. Gerstein, B. Lehner, C. Crombie, J. Tischler, A. Fortunato, A. G. Fraser, M. E. Hillenmeyer, M. J. Dunham, A. C. Gavin, N. J. Krogan, H. Yu, K. Tarassov, J. A. de Visser, C. D. Meiklejohn, D. L. Hartl, A. B. Parsons, and C. S. Sevier. 2010. The genetic landscape of a cell. Science 327:425-31.

Domingo-Calap, P., M. Pereira-Gomez, and R. Sanjuan. 2010. Selection for thermostability can lead to the emergence of mutational robustness in an RNA virus. J. Evol. Biol. 23:24532460 . 
Eyre-Walker, a, and P. Keightley. 2007. The distribution of fitness effects of new mutations. Nat. Rev. Genet. 8:610-8.

Goldsmith, M., and D. S. Tawfik. 2009. Potential role of phenotypic mutations in the evolution of protein expression and stability. Proc. Natl. Acad. Sci. U. S. A. 106:6197-202.

Goyal, S., D. J. Balick, E. R. Jerison, R. A. Neher, B. I. Shraiman, and M. M. Desai. 2012. Dynamic mutation-selection balance as an evolutionary attractor. Genetics 191:1309-1319.

Haigh, J. 1978. The accumulation of deleterious genes in a population-Muller's Ratchet. Theor. Popul. Biol. 14:251-267.

Haldane, J. B. S. 1937. The effect of Variation on Fitness. Am. Nat. LXXI:337-349.

Hermisson, J., and P. Wagner. 2004. The Population Genetic Theory of Hidden Variation and Genetic Robustness. Genetics 168:2271-2284.

Khan, A. I., D. M. Dinh, D. Schneider, R. E. Lenski, and T. F. Cooper. 2011. Negative epistasis between beneficial mutations in an evolving bacterial population. Science (80-. ). 332:1193-1196.

Kimura, M. 1969. The number of heterozygous nucleotide sites maintained in a finite population due to steady flux of mutations. Genetics 61:893-903.

Kimura, M., and T. Maruyama. 1966. The mutational load with epistatic gene interactions in fitness. Genetics 54:1337-1351.

Kondrashov, A. S. 1994. Muller's ratchet under epistatic selection. Genetics 136:1469-1473.

Krakauer, D. C., and J. B. Plotkin. 2001. Redundancy, antiredundancy, and the robustness of genomes. Proc. Natl. Acad. Sci. U. S. A. 99:1405-1409.

Labar, T., and C. Adami. 2016. Evolution of Drift Robustness in Small Populations of Digital Organisms. bioRxiv 1-17.

Lynch, M., D. Butcher, R. Bürger, and W. Gabriel. 1993. The mutational meltdown in asexual populations. J. Hered. 84:339-344.

Meiklejohn, C. D., and D. L. Hartl. 2002. A single mode of canalization. Trends Ecol. Evol. 17:468-473.

Muller, H. J. 1964. The relation of recombination to mutational advance. Mutat. Res. Mol. Mech. Mutagen. 1:2-9.

O'Fallon, B. D., F. R. Adler, and S. R. Proulx. 2007. Quasi-species evolution in subdivided populations favours maximally deleterious mutations. Proc. Biol. Sci. 274:3159-3164.

Ofria, C., D. M. Bryson, and C. O. Wilke. 2009. Avida: A software platform for research in computational evolutionary biology. Pp. 3-35 in Artificial Life Models in Software (Second Edition).

Poon, A., and L. Chao. 2005. The rate of compensatory mutation in the DNA bacteriophage $\square$ ??X174. Genetics 170:989-999.

Poon, a, and S. P. Otto. 2000. Compensating for our load of mutations: freezing the meltdown of small populations. Evolution (N. Y). 54:1467-1479.

Proulx, S. R., and P. C. Phillips. 2005. The Opportunity for Canalization and the Evolution of Genetic Networks. Am. Nat. 165:147-162.

Sanjuan, R., J. M. Cuevas, V. Furio, E. C. Holmes, and A. Moya. 2007. Selection for Robustness in Mutagenized RNA Viruses. Plos Genet. 3:e93.

Schultz, S. T., and M. Lynch. 1997. Mutation and Extinction $\square$ : The Role of Variable Mutational Effects, Synergistic Epistasis, Beneficial Mutations, and Degree of Outcrossing. Evolution (N. Y). 51:1363-1371.

Siegal, M. L., and J.-Y. Leu. 2014. On the Nature and Evolutionary Impact of Phenotypic 

Robustness Mechanisms. Annu Rev Ecol Evol Syst. 45:496-517.

Silander, O. K., O. Tenaillon, and L. Chao. 2007. Understanding the evolutionary fate of finite populations: The dynamics of mutational effects. PLoS Biol. 5:922-931.

Steinberg, B., and M. Ostermeier. 2016. Shifting Fitness and Epistatic Landscapes Reflect Tradeoffs along an Evolutionary Pathway. J. Mol. Biol. 428:2730-2743. Elsevier Ltd.

Szollosi, G. J., and I. Derenyi. 2007. Congruent Evolution of Genetic and Environmental Robustness in Micro-RNA. Mol. Biol. Evol. 26:867-874.

van Nimwegen, E., J. P. Crutchfield, and M. Huynen. 1999. Neutral evolution of mutational robustness. Proc. Natl. Acad. Sci. U. S. A. 96:9716-9720.

Visser, J. A. G. M., J. Hermisson, G. P. Wagner, L. A. Meyers, H. Bagheri-Chaichian, J. L. Blanchard, L. Chao, J. M. Cheverud, S. F. Elena, W. Fontana, G. Gibson, T. F. Hansen, D. Krakauer, R. C. Lewontin, C. Ofria, S. H. Rice, G. von Dassow, A. Wagner, and M. C. Whitlock. 2003. Perspective: Evolution and Detection of Genetic Robustness. Evolution (N. Y). 57:1959-1972.

Wager, G. P., and W. Gabriel. 1990. Quantitative Variation in Finite Parthenogenetic Populations: What Stops Muller's Ratchet. Evolution (N. Y). 44:715-731.

Wagner, A. 2013. Robustness and evolvability in living systems.

Wagner, G. P., G. Booth, H. Bagheri-chaichian, G. P. Wagner, G. Booth, and H. Bagherichaichian. 1997. A Population Genetic Theory of Canalization. Evolution (N. Y). 51:329347.

Wilke, C. O. 2001. Adaptive Evolution on Neutral Networks. 715-730.

Wilke, C. O., and C. Adami. 2003. Evolution of mutational robustness.

Wilke, C. O., J. L. Wang, C. Ofria, R. E. Lenski, and C. Adami. 2001. Evolution of digital organisms at high mutation rates leads to survival of the flattest. Nature 412:331-333.

Wylie, C. S., and E. I. Shakhnovich. 2011. A biophysical protein folding model accounts for most mutational fitness effects in viruses. Proc. Natl. Acad. Sci. U. S. A. 108:9916-21.

611

612 
613 Figure 1: Under a fixed ratio of beneficial to deleterious mutation rates, populations residing on

614 steeper fitness landscapes survive under higher deleterious mutation rate

615 A. Equilibrium fitness of finite populations under different deleterious mutation rates $\left(U_{d e l}\right)$ on

616 fitness landscapes with two different selection coefficients $(s)$. Dashed black line: theoretical

617 prediction for equilibrium fitness in infinite populations $\left(e^{-U_{d e l}}\right)$. Populations on the steeper

618 fitness landscape (blue), i.e., the one with a larger s, maintain MSDE under higher $U_{d e l}$ than do

619 populations on the shallower landscape (red). Beneficial mutation rate $U_{b e n}=0.01 U_{d e l}$.

620 Population size $N=1000$. Average fitness is recorded after evolving for 10,000 generations

621 (see Methods). Error bars: standard deviation over 50 replicates. Inset: comparison of the two

622 fitness landscapes, each of which is composed of one isotropic peak without epistasis.

623 B. Critical $U_{d e l}$ values from simulations (points) and analysis (lines, derived numerically from

624 Eqn. 7 in Goyal et al. 2012), for landscapes with different selection coefficients in the absence of

625 epistasis. Error bars: uncertainty associated with locating the critical $U_{d e l}$ from simulation results

626 (see Methods). Regardless of population size, populations on steeper landscapes always survive

627 under higher $U_{\text {del }}$.

628 
629 Figure 2: Fitness landscapes with negative epistasis protect populations from Muller's ratchet

630 while ones with positive epistasis make populations vulnerable to the ratchet

631 A. Cartoon of evolutionary dynamics of populations on fitness landscape composed of one

632 isotropic peak with negative epistasis. Curved dashed line: fitness landscape. Straight tangent

633 dashed line: fitness landscape without epistasis that shares the same selection coefficient at the

634 peak. Note that the selection coefficient (the slope of the fitness landscape) increases with the

635 number of deleterious mutations. Golden vertical line and horizontal dashed line: stable MSDE

636 at the peak $e^{-U_{d e l}}$ (see main text, also S1 Text).

637 B. Time course of average number of deleterious mutations in 35 populations initialized at

638 random points on isotropic fitness peak with negative epistasis during 10,000 generations

$639\left(N=1000, s=0.2, U_{d e l}=1.0, U_{b e n}=0.01 U_{d e l}, \varepsilon=-0.25\right)$. Golden vertical line: same as

640 in A. All populations converge to the MSDE regardless of where they are initiated (green traces).

641 C. Cartoon of evolutionary dynamics of populations on fitness landscape composed of one

642 isotropic peak with positive epistasis. Curved dashed line: fitness landscape. Straight tangent

643 dashed line: fitness landscape without epistasis that shares the same selection coefficient at the

644 peak. Note that selection coefficient (equal to the slope of the fitness landscape) decreases with

645 the number of deleterious mutations. Cyan vertical line and horizontal dashed line: unstable

646 MSDE at the peak $e^{-U_{\text {del }}}$ (see main text). Violet vertical line: predicted point of no return

647 (derived numerically from Eqn.7 in Goyal et al. 2012).

648 D. Time course of average number of deleterious mutations in 100 populations initialized at

649 random points on an isotropic fitness peak with positive epistasis during 10,000 generations

$650\left(N=1000, s=0.5, U_{d e l}=0.5, U_{b e n}=0.01 U_{d e l}, \varepsilon=0.25\right)$. Cyan and violet vertical lines:

651 same as in C. Populations initiated above the point of no return tend to evolve to the peak (blue 
652 traces), whereas ones initialized below it tend to succumb to Muller's ratchet (pink traces).

653 Realizations that fluctuate across the point of no return are colored in brighter blue and pink.

654 
655 Figure 3: Populations converge to peak with negative epistasis on multi-peak fitness landscape

656 A. Cartoon of evolutionary dynamics of populations on multi-peak fitness landscape. Both

657 fitness peaks are isotropic, one with only positive epistasis (left) and the other with only negative

658 epistasis (right). Curved dashed lines on both sides represent the fitness landscape. Straight

659 tangent dashed line on both sides again represent the slope (i.e. the selection coefficient at the

660 peaks); this quantity is equal at both peaks. Horizontal dashed line: $e^{-U_{d e l}}$ on both sides. Cyan

661 vertical line: $e^{-U_{d e l}}$ on the positive epistasis side. Violet vertical line: numerically derived point

662 of no return on the positive epistasis side (Eqn.7 in Goyal et al. 2012). Black vertical line: valley

663 of the landscape. Golden vertical line: $e^{-U_{d e l}}$ on the negative epistasis side.

664 B. Time course of average number of deleterious mutations in 100 populations initiated at

665 random, uniformly distributed points on multi-peak landscape over the course of 10,000

666 generations $\left(N=10,000, s=0.3, U_{d e l}=0.5, U_{b e n}=0.01 U_{d e l}\right.$, positive epistasis side

$667 \varepsilon=0.25$, negative epistasis side $\varepsilon=-0.25)$. Cyan, violet, black and golden vertical lines:

668 same as in B. Populations are color coded based on their starting point (blue traces: above the

669 point of no return on the positive epistasis side, pink traces: below the point of no return on the

670 positive epistasis side, bright pink and blue traces: realizations that fluctuate across the point of

671 no return on the positive epistasis side, green traces: populations initialized on the negative

672 epistasis side). The vast majority of populations below the point of no return rapidly cross the

673 valley and converge to MSDE on the negative epistasis side. Due to uniformly stronger selection

674 on the negative epistasis side of the valley, populations there exhibit less stochasticity in their

675 trajectories. Simulations in smaller population size $(N=1000)$ show qualitatively the same

676 results, although it takes much longer to converge to the negative epistasis side (data not shown). 
678 Figure 4: Mutational robustness and ratchet robustness on hybrid peaks

679 A. Cross-section through a hybrid fitness peak. While all first mutations in the highest fitness

680 genotype share the same fitness effect, a fraction $p$ of them cause subsequent mutations to

681 exhibit positive pairwise epistasis, and the remaining fraction $1-p$ cause subsequent mutations

682 to exhibit negative epistasis. Populations are always initiated at the peak. Here $p=0.5$.

683 B. Equilibrium proportions of individuals at the peak (black), in the negative epistasis region

684 (blue), and in the positive epistasis region (pink), as a function of $U_{d e l}\left(U_{b e n}=0.01 U_{d e l}\right.$,

$685 p=0.5, N=1000, s=0.35$, negative epistasis region: $\varepsilon=-0.25$, positive epistasis

686 region: $\varepsilon=0.25$ ), evolved for 10,000 generations, error bars: standard deviation across 50

687 replicates. Under $U_{d e l}$ less than the critical $U_{d e l}$ at the peak (here $\sim 1.1$; see main text), the

688 subpopulation on the negative epistasis region relies on continual mutational input from

689 subpopulation on the peak. After $U_{d e l}$ exceeds the critical $U_{d e l}$ at the peak, the subpopulation on

690 the peak goes extinct, and with it, the subpopulation on the negative epistasis region. At this

691 point, the remaining population finds itself beyond the point of no return on the positive epistasis

692 region (see main text), and it succumbs to Muller's ratchet.

693 C. Equilibrium proportions of individuals at the peak (black), the negative epistasis region (blue),

694 and the positive epistasis region (pink), as a function of $U_{d e l}$ (parameters are exactly the same as

695 panel B except $p=0.01$ ), evolved for 10,000 generations, error bars: standard deviation across

69650 replicates. Population dynamics resemble $p=0.5$ (see main text). 
698 Figure 5: Increasing $U_{b e n} / U_{d e l}$ with decreasing fitness protects populations from Muller's

699 ratchet

700 Equilibrium fitness as a function of total mutation rates $(U)$ in finite asexual populations with

701 evolving and fixed $U_{b e n} / U_{d e l}$. Dashed black line: theoretical prediction for equilibrium fitness in

702 infinite populations $\left(e^{-U_{d e l}}\right)$ with fixed $U_{b e n} / U_{d e l}\left(U_{b e n}=0.01 U_{d e l}\right)$. Population size $N=$

703 1000. Selection coefficient $s=0.4$. Average fitness is recorded after evolving for 10,000

704 generations (see Methods). Populations in which $U_{b e n} / U_{d e l}$ evolve by virtue of reversion

705 mutations (blue) survive under high $U$, while ones with fixed $U_{b e n} / U_{d e l}$ (red) succumb to

706 Muller's ratchet. (These latter results are identical to those shown for $s=0.4$ in Fig. 1.) Error

707 bars: standard deviation over 50 replicates.

708 
709 Figure 6: Annotated Fig. 2 from Wilke et al. (2001) suggests the evolution of ratchet robustness

710 in populations of digital organisms. Populations in Avida (Ofria et al. 2009) were evolved for

7111,000 generations at low and high mutation rates $(\mu=0.5$ and 2.0 , respectively). In this figure,

712 the fitness landscapes in the mutational neighborhoods of these two populations were examined

713 by propagating new populations seeded with one representative individual from each for 15

714 generations. Top panels: "A" designates new populations seeded with a representative individual

715 adapted under low mutation rate. Bottom panels: "B" designates new populations seeded with a

716 representative individual adapted under high mutation rate. $\mu$ denotes the mutation rate during

717 the subsequent 15 generation experiment. Each bar indicates the frequency of genotypes with the

718 corresponding number of mutations at the end of these 15 generations; colors represent the

719 fitness of these genotypes. The left two panels illustrate the fitness landscape in the nearby

720 mutational neighborhood of A or B (small $\mu=0.5$ ). Importantly, the descendants of the

721 individual from the B population include many more lethal mutants (black) than do those from

722 the individual from the A population (red arrows). The right two panels depict the fitness

723 landscape across a broader mutational neighborhood of A or B (large $\mu=2.0$ ). Notably, the

724 viable descendants of the individual from the B population carry fewer deleterious mutations

725 than do those from the A population (red dashed lines represent the approximate mutational limit

726 among viable offspring: upper panel skews towards more mutations than does the lower panel).

727 Both observations suggest that selection against deleterious mutation is stronger in the

728 mutational neighborhood occupied by the B population than it is in the neighborhood of the A

729 population, consistent with the evolution of ratchet robustness rather than mutational robustness

730 in response to elevated mutation rate. 
A

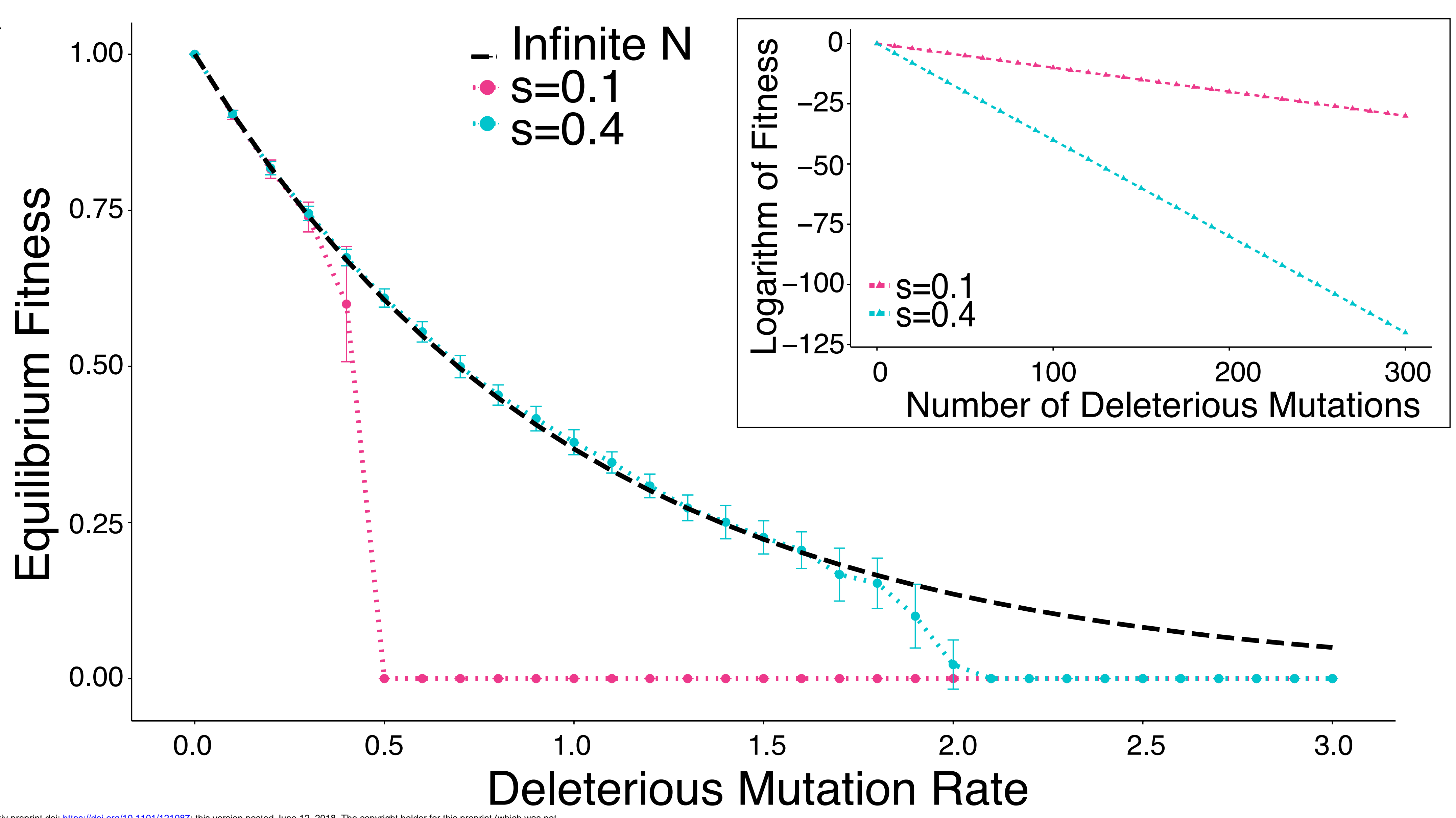

B

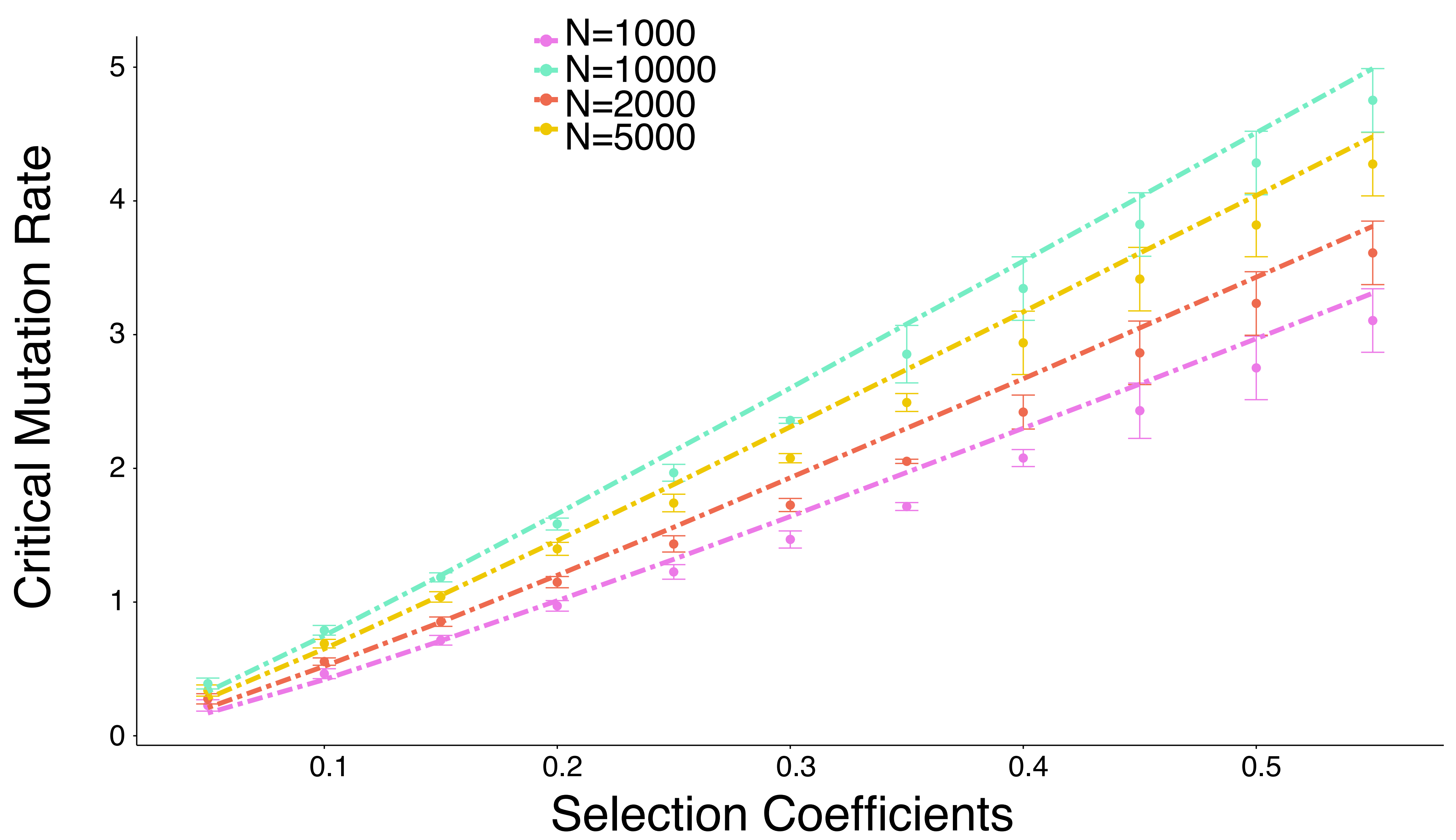


A

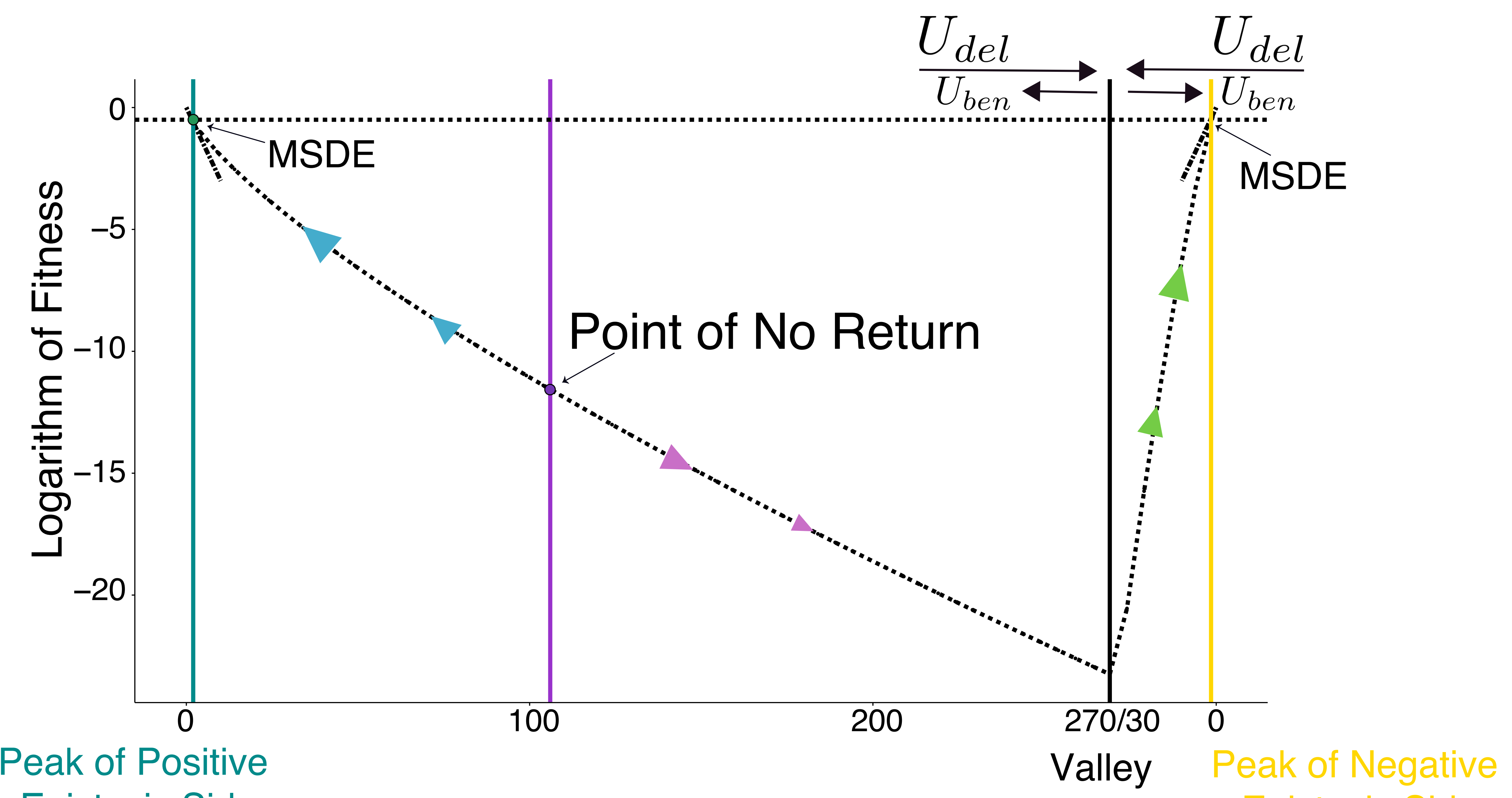

Epistasis Side

Number of Deleterious Mutations From Corresponding Peak

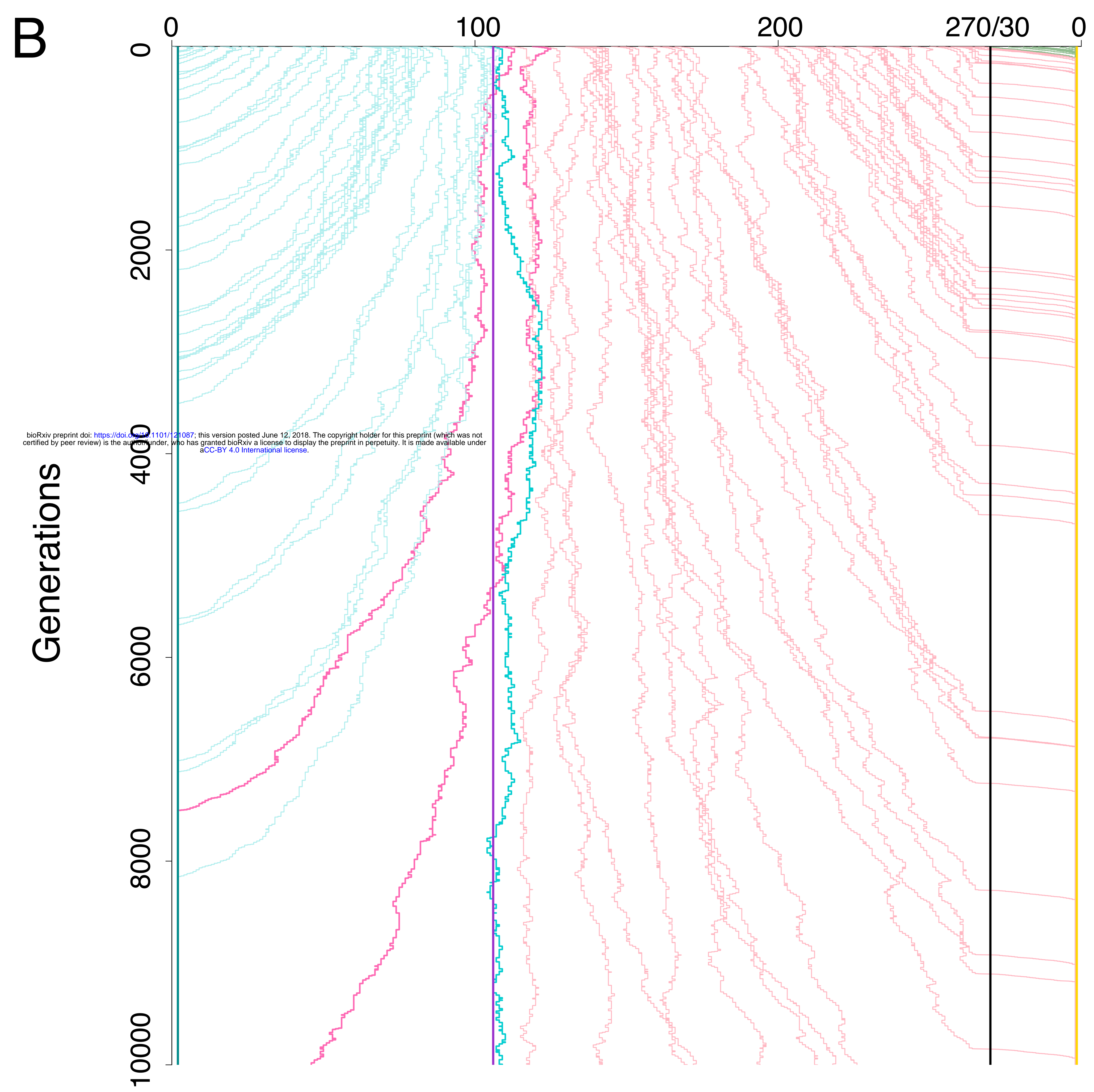



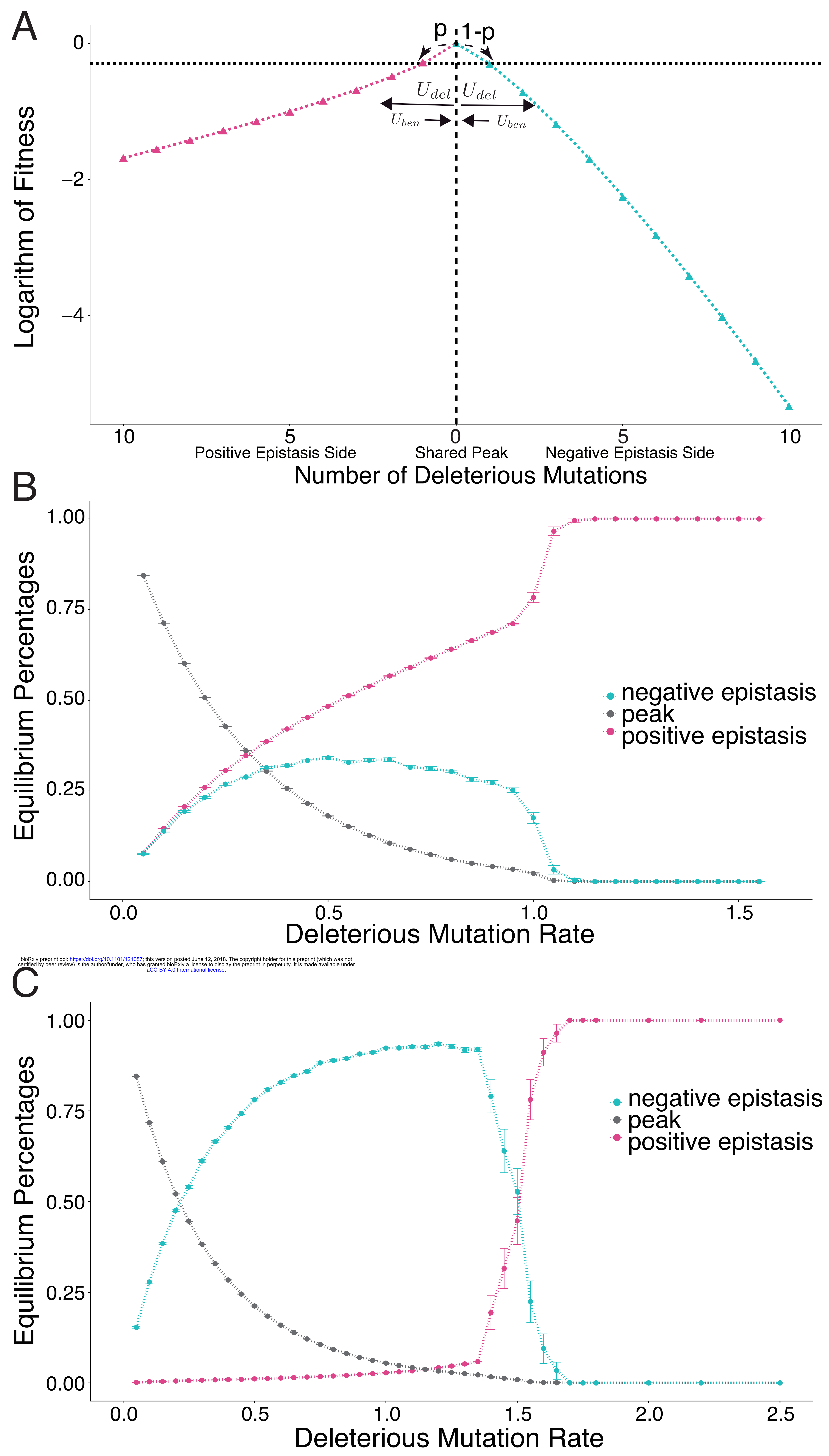


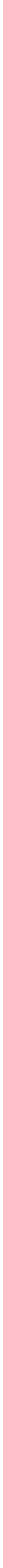




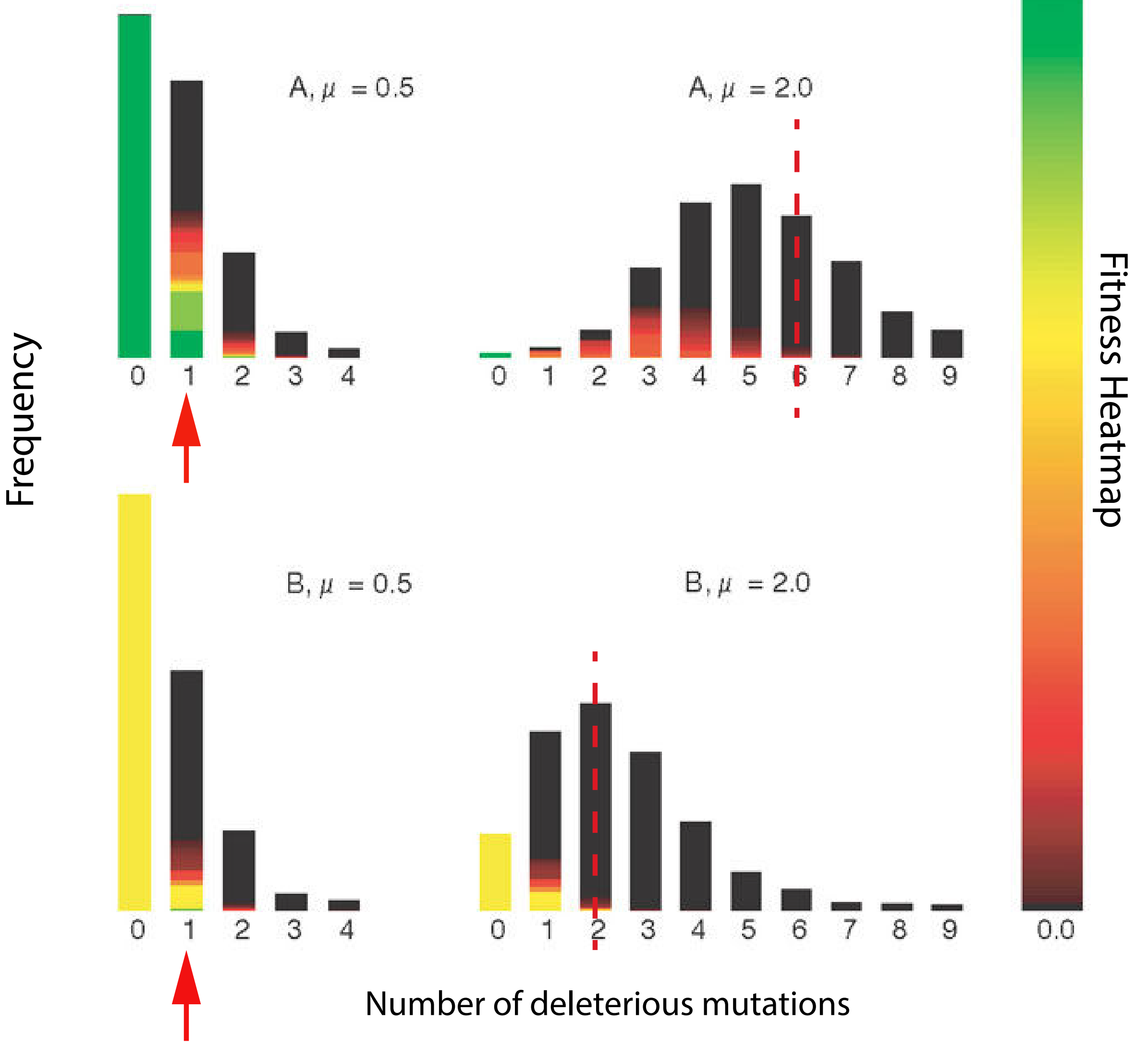

